\title{
Reconfigurable Stimulus Generator for Receive Beamformer Test-Bed
}

\author{
Khyati R.Zalawadia \\ BITs educampus \\ Vadodara India
}

\author{
Neeraj Mishra \\ Space Applications Centre \\ ISRO,Ahmedabad India
}

\author{
Himanshu Shah \\ Space Applications Centre \\ ISRO,Ahmedabad India
}

\author{
U.D.Dalal \\ SardarVallbhbhai National Institute of Technology, \\ Surat -395007 India
}

\begin{abstract}
Laboratory testing of digital beamforming processor is required to test and optimize the beamformer algorithm, code and hardware before integrating with antenna and testing the beamformer in an anechoic chamber. Development of a stimulus generator, for carrying out such an exercise, itself can be quite an involved task for a digital beamformer employing a large phased array antenna. This paper proposes an innovative design of a stimulus generator for the receive beamformer system. A reconfigurable hardware prototype, with a Xilinx Virtex-5 FPGA based architecture, is developed to generate IF stimulus for an 8-elements receive beamformer. It can provide a stimulus for upto four transmitting sources, each with programmable frequency, bandwidth, direction of arrival, S/N and interference. The beamformer performance testing for selective element failure can also be facilitated. Testing of a beamformer with larger phased array antenna can be achieved using multiple cards. The hardware test results are reported
\end{abstract}

\section{General Terms}

Digital Beam Former Stimulus Generator

\section{Keywords}

Digital beam former, Generator, Phased array antenna, Test bed, Xilinx Virtex-5 FPGA

\section{INTRODUCTION}

Phased array antenna (PAA) with beamforming-based architectures enhances the performance of satellite communication systems by improving capacity, coverage, and throughput and provides improvement in QoS while reducing the terminal size [1].

Digital beamforming system consists of phased array antenna and digital beam forming processor. The performance of the antenna system and digital hardware should verify separately before their final integration. The development of a beamformer imposes challenging demand on test and evaluation system. One such demand is to generate the simultaneous signals for all antenna array sensors superimposed with impairments as seen by the antenna system. Impairment may be noise and/or interference signal. These generated signals are use for characterizing the digital beamforming processor before antenna integration.

The recent advances in FPGA technology makes architecture of PAA stimulus generator based on FPGA an attractive option. Few examples, MIMO systems, which is utilize multiple FPGA's and DSP's as discussed in [2]-[5]. Madani et al. have described anantenna simulator, developed under a contract from European Space Agency (ESA), to evaluate the performance of an Adaptive Digital Beam Forming Network [6]. Their work is on MMIC based analog phase shifter approach. Taco Kluwer et el have reported the development of smart antenna test-bed for software defined digital beamforming [7]. Heung-Jae Im have performed analysis of the smart antenna system operating in a wide-band CDMA WLL channel through a test-bed implemented on a DSP board [8]. Further M.Sc. thesis of Juan A. Torres-Rosario discusses the development of test set-up, based on Lyrtech VHS-DAC high-speed multichannel development platform, to test a 16 channels receive beam former [9]. However, developed test-bed is not configurable of generating channel impairments and channel noise.

With the recent trend of satellites with large number of spot beams, the complexity of test-bed required for performance testing of onboard digital hardware of digital beam former have increased multi-fold. In this paper, a design of PAA stimulus generator, which allows one to test a digital beamforming processor for a variety of parameters are reported; thus providing a flexible and versatile solution for testing of a beamforming processor. The developed hardware output mimics the inputs at the phased array antenna module at IF level. The signals fed to IF ports of receiver section to characterize the digital beam forming at IF level.

Section 2 briefly presents the theory of phased array antenna. Section 3 describes the requirements and specifications for phased array antenna stimulus generator. Section 4 describes system architecture of phased array antenna stimulus generator. Section 5 presents the results.

\section{PHASE ARRAY ANTENNATHEORY}

Beamforming adjusts the gain and phase of signal for each antenna array element to compensate for the different delays and attenuations associated with signal paths to elements to increase the signal quality in specific direction of arrival.

Here only narrowband signal is considered. If the bandwidth $\mathrm{B}_{\mathrm{s}}$ of the modulated signal is small, compared to the carrier's frequency, then it is consider a narrowband signal. Another way to define a beamformer signal to be narrowband is:

$\Delta t_{\max } * B_{s} \ll 1$

where, $\Delta t_{\max }$ is the maximum travel time of a plane wave between any two elements of the array.

Let $\tilde{x}(t)$ represent the complex modulated narrowband signal, which needs to be transmitted. To generate the signal for each sensor, the modulated signal should be applied with precise phase delay $\theta_{k}$ corresponding to the $\mathrm{k}^{\text {th }}$ sensor in the array. This is done by applying complex weights $\omega_{k}$ to the signal for $\mathrm{k}^{\text {th }}$ sensor, where, 
$\omega_{k}=A_{k} e^{j \theta_{k}}$

$\mathrm{k}=0,1,2, \ldots, \mathrm{K}-1$,

$\mathrm{K}=$ number of phased array elements.

The resulting output signals for phased array antenna stimulus generator are given by:

$\tilde{y}_{k}(t)=\tilde{x}(t) \omega_{k}$

\subsection{Stimulus Generation for Linear Antenna Geometry}

For a linear array geometry (Figure 1) with element spacing d, the received signal for the $\mathrm{k}^{\text {th }}$ sensor of the PAA contains the time delay $\tau_{k}$, called steering time delay. This steering delay $\tau_{k}$ with respect to reference element is given by:

$\tau_{k}=k \sin (\theta) \frac{d}{c}$

$\mathrm{k}=0,1, \ldots, \mathrm{K}-1 ; \quad \mathrm{K}=$ No. of phased array elements

$\mathrm{c}=$ velocity of EM waves in the medium

The corresponding phase delay $\theta_{k}$ for a narrowband signal with carrier frequency $f c$ is given by:

$\theta_{k}=2 \pi f_{c} \tau_{k}$

\subsection{Stimulus Generation for Rectangular Antenna Geometry}

For a rectangular antenna array geometry (Figure 2), where $\mathrm{M}$ elements are arranged in the $\mathrm{x}$ direction and $\mathrm{N}$ elements are arranged in y direction and the inter-element spacing is $\mathrm{dx}$ and dy in $\mathrm{x}$ and $\mathrm{y}$ direction respectively, the steering delay $\tau_{(\mathrm{m}, \mathrm{n})}$ with respect to reference element at $(0,0)$ is given by:

$\tau_{(m, n)}=\left(\frac{\mathrm{md}_{\mathrm{x}}}{\mathrm{c}} \cos (\varphi) \sin (\theta)+\frac{n d_{y}}{c} \sin (\varphi) \sin (\theta)\right)$

where, $\mathrm{m}=0,1, \ldots, \mathrm{M}-1$ and $\mathrm{n}=0,1, \ldots, \mathrm{N}-1$.

The corresponding phase delay $\theta_{(m . n)}$ for a narrowband signal with carrier frequency fc is given by:

$\theta_{(m . n)}=2 \pi f_{c} \tau_{(m, n)}$

\subsection{Stimulus Generation for Triangular Antenna Geometry}

If alternate samples removed in rectangular antenna geometry, a triangular phase array antenna shown in Figure 3 obtained. A triangular grid is more efficient for suppressing grating lobes than a rectangular grid; and for a given aperture size, fewer elements are required. (6) and (7) respectively obtain the steering delay and phase delay for atriangular geometry, where only alternate values of $\mathrm{m}$ and $\mathrm{n}$ are used. i.e. $\mathrm{m}=0,2$, $4, \ldots, \mathrm{M}-1$ and $\mathrm{n}=0,2,4, \ldots, \mathrm{N}-1$.

Similarly, various other sensor arrangements are possible for constructing a phased array system and corresponding equation for phase delay obtained.

The stimulus in each case can be generated using (3).

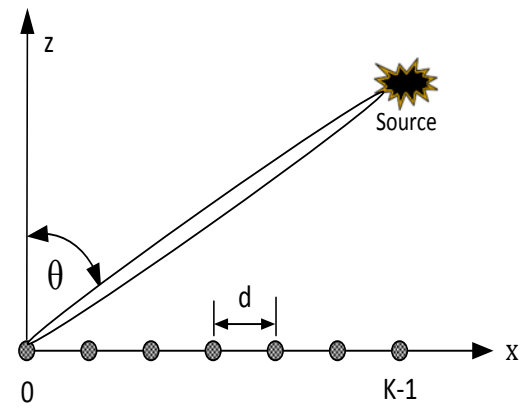

Figure 1: Linear antenna geometry

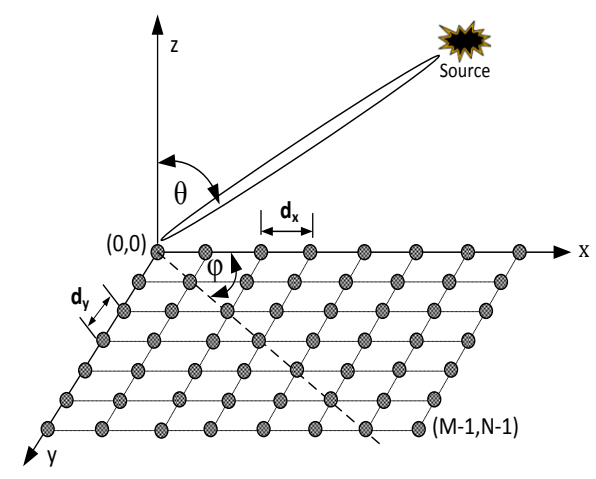

Figure. 2: Rectangular antenna geometry

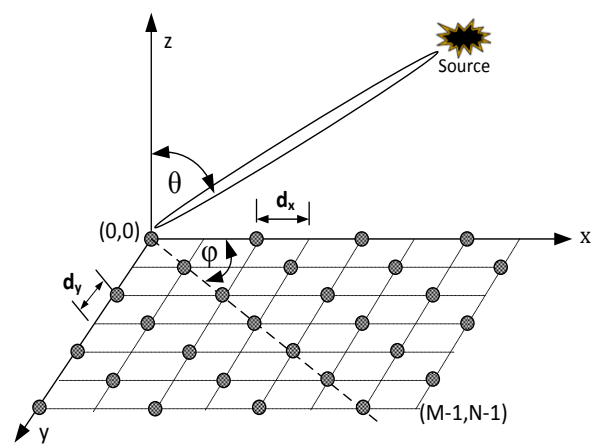

Figure. 3: Triangular antenna geometry

\section{REQUIREMENT and SPECIFICATION}

Broad requirements for the stimulus generator summarized below:

- To generate signals for large number of array elements with re-configurable geometry

- To generate a signal for a source from any direction

- To generate multi-carrier signals with varying bandwidth and power

- To generate impairment and selective element failure

The specifications for the PAA stimulus generator developed are as given in Table 1 . 
Table 1. Specifications of PAA Stimulus Generator

\begin{tabular}{|c|c|}
\hline Parameter & Specification \\
\hline Output frequency band & $70 \pm 18 \mathrm{MHz}$ \\
\hline Output power level & 0 to $-20 \mathrm{dBm}$ \\
\hline Output impedance & 50 \\
\hline No. of elements & Upto 8 with single card \\
More than 8 with multiple cards
\end{tabular}

\section{DESIGN OF PAA STIMULUS \\ GENERATOR}

Figure 4 shows the architecture of a conventional stimulus generator for receive beamformer. It takes an RF source as input and generates multiple sources via a power divider network. Then variable phase shift and attenuation to each source apply for a particular direction of incidence. For multiple beams, multiple hardware are required to be implemented with a combiner for each element as shown in Figure 5. It is obvious that the large RF hardware needed.

Figure 6 describes the block diagram of the Xilinx Virtex-5 FPGA based stimulus generator for receive beamformer testbed. The FPGA based design not only saves large RF hardware, it provides programmability of test parameters also which is quite useful for testing the receive beamformer.

The advantages of FPGA based stimulus generator summarized as follows:

- No need of RF source. RF signal generation in FPGA.

- Generation of multi-beam signals with programmable frequency, bandwidth, power and DOA

- Scanning of beam to facilitate pattern measurement

- Cost saving and less hardware requirement

- A PC based controller provides convenient GUI for programming of the stimulus generator

The FPGA design divided into three basic modules: Signal Generator and Weighting Module, Summing and Preemphasis Module and MIL-STD-1553B Interface Module.

\subsection{Signal Generator and Weighting Module}

This module consists of pseudo random generator, BPSK modulator and complex weight multiplier for 8 PAA elements. Programmability for centre frequency, bandwidth and modulation on/off provided. Four numbers of such modules are required for generation of four carriers.

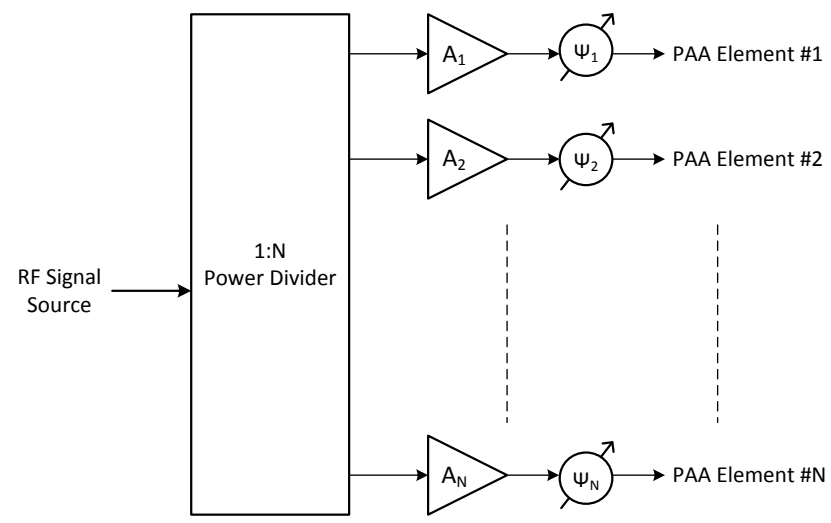

Figure 4: Conventional stimulus generator for receive beamformer (1 Beam)
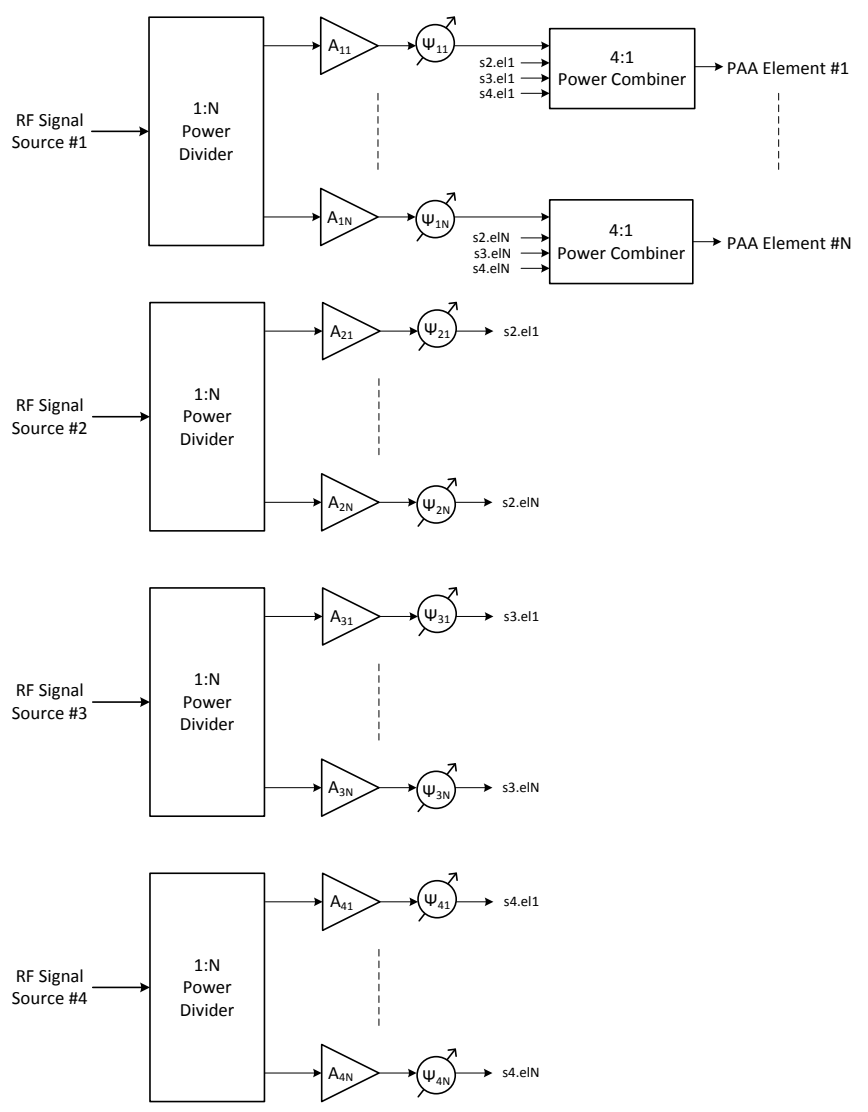

Figure 5: Conventional stimulus generator for receive beamformer (4 Beam)

Each carrier can emulate beam coming from different direction. Multiple cards can synchronize to maintain signal coherence for large PAA. 


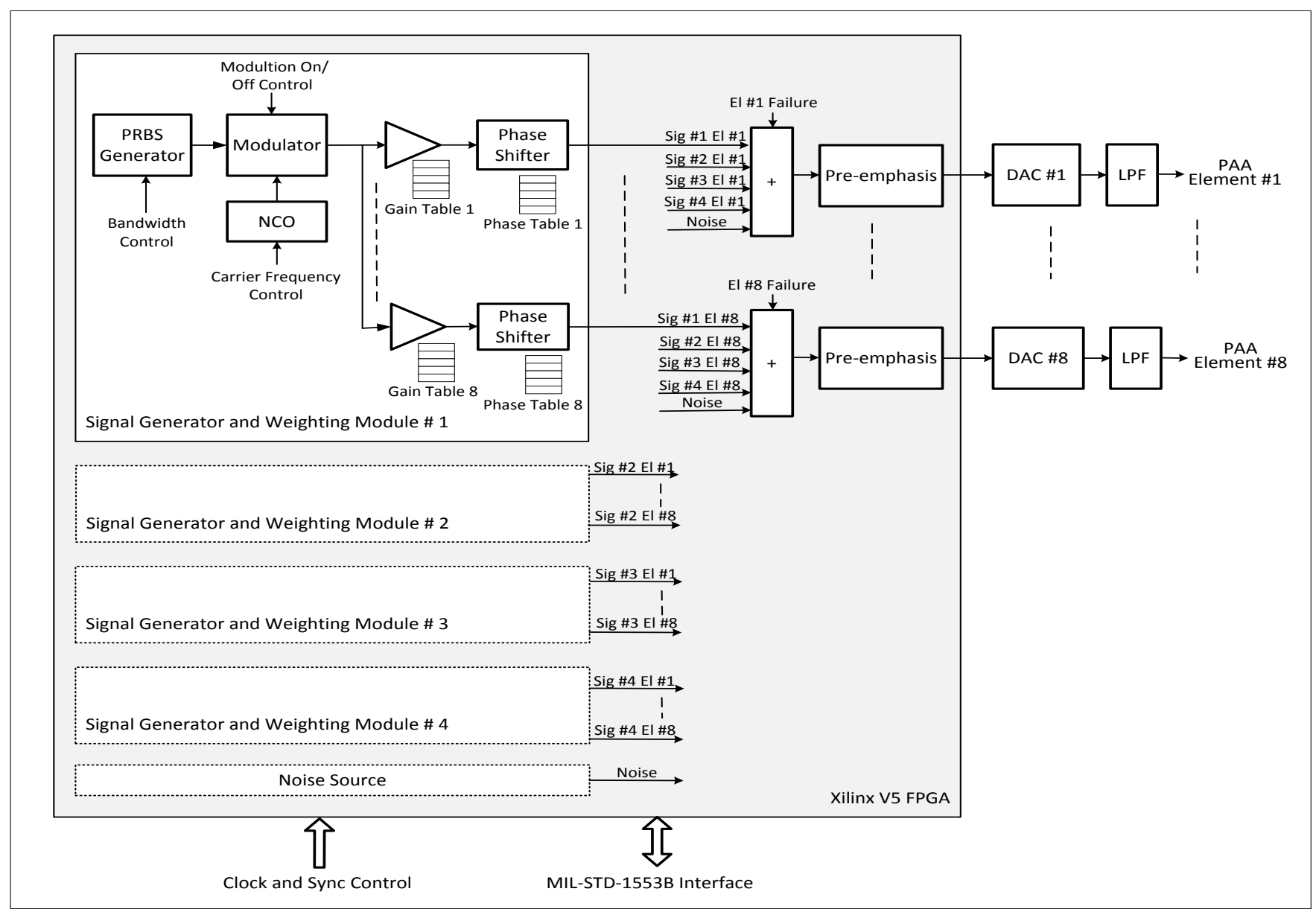

Figure 6: FPGA implementation of phased array antenna stimulus generator

\subsection{Summing and Pre-emphasis Module}

The corresponding outputs from four Signal Generator and Weighting Modules added to generate signal for each PAA element. Then a pre-emphasis filter boosts the high frequencies as per $\mathrm{x} / \sin (\mathrm{x})$ response to compensate the effect of DAC's $\sin (\mathrm{x}) / \mathrm{x}$. Programmability for setting $\mathrm{S} / \mathrm{N}$ and selective PAA element failure provided. Total 8 Summing and Pre-emphasis Modules produce signals corresponding to 8 PAA elements.

\subsection{MIL-STD-1553B Interface Module}

This module implements remote terminal in the Stimulus generator and allows configuring the look up tables for gain and phasing delay. As well as to control bandwidth, centre frequency, power, $\mathrm{S} / \mathrm{N}$ and impairments for each source signal using a PC based controller, which provides convenient GUI for entry of look angles as well as parameters related to each source signal to be generated.

\section{SIMULATION AND HARDWARE RESULTS}

The stimulus generator designed for a beamformer with PAA geometry as specified in Table 2. Figure 7 and 8 shows the Matlab simulation results for azimuth and elevation respectively corresponding to four signal sources located as shown in Table 3.

The stimulus generator was thoroughly tested in lab. Table 4 shows the target specification and measured results for stimulus generator.
Table 2. Beamformer Geometry

\begin{tabular}{|c|c|}
\hline Parameter & Specification \\
\hline Geometry & Triangular \\
\hline No. of element & 30 \\
\hline Element spacing & $\mathrm{dx}=1.67 \lambda, \mathrm{dy}=2.67 \lambda$ \\
\hline Frequency Band & $2.67 \mathrm{GHz}$ \\
\hline
\end{tabular}

Table 3. Signal Locations

\begin{tabular}{|c|c|c|}
\hline & Azimuth & Elevation \\
\hline Source \#1 & $65^{\circ}$ & $30^{\circ}$ \\
\hline Source \#2 & $31^{\circ}$ & $50^{\circ}$ \\
\hline Source \#3 & $110^{\circ}$ & $60^{\circ}$ \\
\hline Source \#4 & $140^{\circ}$ & $72^{\circ}$ \\
\hline
\end{tabular}

This simulator was used in testing of 4-element PAA digital hardware of receive digital beam former (DBF), a technology development program in Space Applications Centre (ISRO) India. 
The stimulus generator produced the 4 PAA signals with different DOAs in $70 \pm 18 \mathrm{MHz}$ frequency band with dynamic channel loading. Figure 9, 10 and 11 show the polar plots of hardware test result for DBF linear phase phased array configuration at DOA angles $\pm 30^{\circ}$ and $60^{\circ}$ generated by stimulus generator.

\section{Table 4.Measured Result of Reconfigurable Stimulus} Generator

\begin{tabular}{|c|c|c|}
\hline Parameter & Specification & Measured result \\
\hline $\begin{array}{l}\text { Output } \\
\text { frequency } \\
\text { band }\end{array}$ & $70 \pm 18 \mathrm{MHz}$ & $70 \pm 18 \mathrm{MHz}$ \\
\hline $\begin{array}{c}\text { Output } \\
\text { power level }\end{array}$ & 0 to $-20 \mathrm{dBm}$ & 0 to $-22 \mathrm{dBm}$ \\
\hline $\begin{array}{l}\text { Output } \\
\text { impedance }\end{array}$ & $50 \Omega$ & $50 \Omega$ \\
\hline $\begin{array}{l}\text { No. of } \\
\text { elements }\end{array}$ & $\begin{array}{l}\text { Upto } 8 \text { with single card } \\
\text { More than } 8 \text { with } \\
\text { multiple cards }\end{array}$ & $\begin{array}{l}\text { Only up to } 8 \text { element } \\
\text { in single card }\end{array}$ \\
\hline $\begin{array}{l}\text { No of } \\
\text { sources }\end{array}$ & Upto 4 & Upto 4 \\
\hline $\begin{array}{l}\text { Signal } \\
\text { control }\end{array}$ & 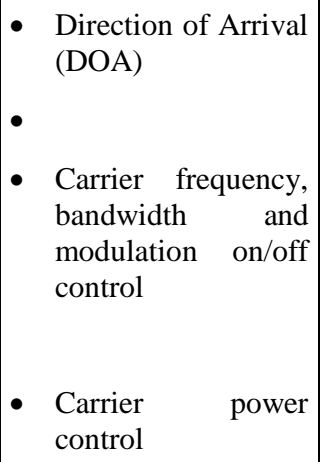 & $\begin{array}{l}\text { - DOA tested with } \\
\text { spacing 0.81 \& } \\
0.5 \lambda \text { spacing } \\
\text { - Carrier frequency, } \\
\text { bandwidth and } \\
\text { modulation on/off } \\
\text { control } \\
\\
\text { - Carrier power } \\
\text { control }\end{array}$ \\
\hline $\begin{array}{l}\text { Other } \\
\text { functions }\end{array}$ & $\begin{array}{c}\text { Scanning of source } \\
\text { beam }\end{array}$ & $\begin{array}{l}\text { Scan test performed } \\
\qquad-65^{\circ} \leq \Theta \leq 65^{\circ}\end{array}$ \\
\hline
\end{tabular}

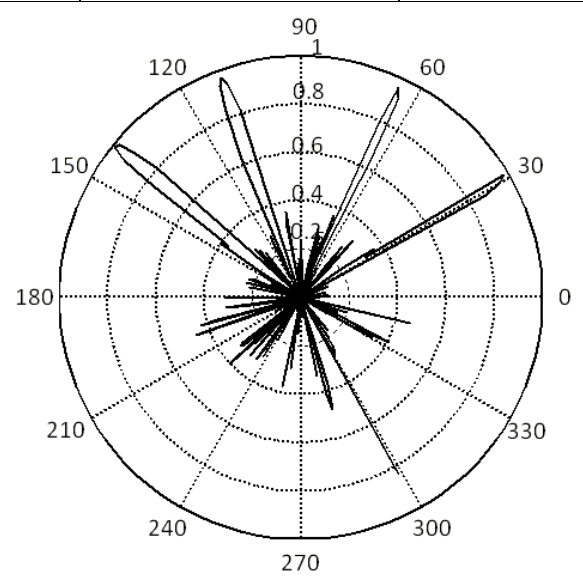

Figure 7: Stimulus Generator Simulation Result for Azimuth

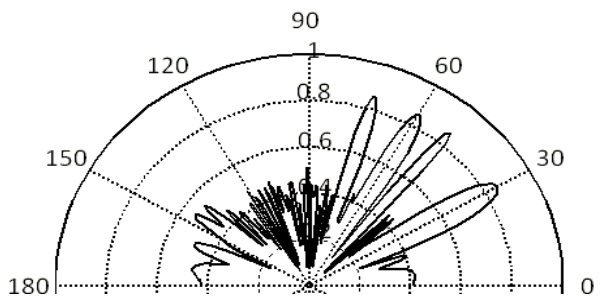

Figure 8: Stimulus Generator Simulation Result for Elevation

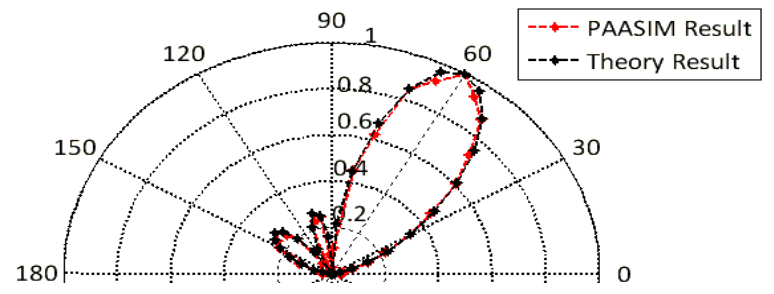

Figure 9: Stimulus Generator Hardware and Simulation Result for linear array $\Theta=30$ 。

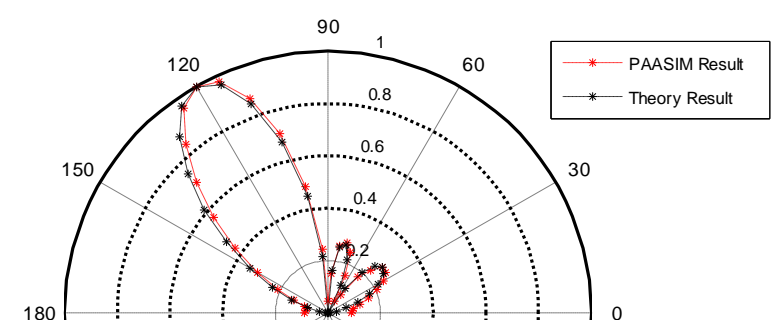

Figure10: Stimulus Generator Hardware and Simulation Result for linear array $\Theta=-30$ 。

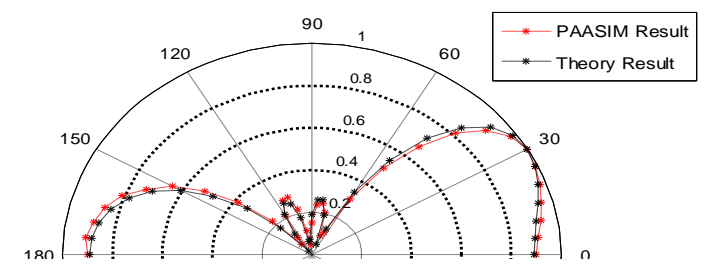

Figure11: Stimulus Generator Hardware and Simulation Result for linear array $\Theta=60$ 。

\section{CONCLUSION}

In this paper, a Xilinx Virtex-5 FPGA based stimulus generator for receive beamformer test-bed is described. It is shown that the implemented design offers a number of advantages like saving of large RF hardware and programmability of frequency, power, DOA, S/N, interference, selective failure of PAA, beam scanning etc. compared to conventional RF hardware based stimulus generator. The stimulus generator has proven to be a versatile tool for testing of digital receives beam former system before integrating it to the actual antenna system.

The system can generate stimulus corresponding to upto 8 PAA elements for upto 4 sources coming from different directions. Multiple such cards can combine to test a beamformer for a large PAA. The developed simulator is able to cope up the increasing demand of multiple source.

The developed simulator has limitation due to implementation of narrow band algorithm. The future scope will be the modification of algorithm for wideband signals generation. 
Further, the effects formutual coupling between the antenna elements can also be incorporated in design to improve the accuracy of stimulus generator.

\section{ACKNOWLEDGMENTS}

The authors wish to acknowledge the contribution of Milind Mahajan of ASG and Pramod, Pratik, Jaydeep, Md. Azhar and Amita Mevada of DTD in this project. The authors also hereby acknowledge the guidance and support provided by Alak Banik (GD), Deepak Das (DD) and A S Kiran Kumar, Director, Space Applications Centre (ISRO).

\section{REFERENCES}

[1] Steven D. Blostein and Harry Leib, "Multiple antenna systems: Their role and impact in future wireless access", IEEE Communication. Mag.,vol.41, no.7, pg.94-101, 2003

[2] W. Xiang, P. Richardson, B. Walkenhorst, X. Wang, and T. Pratt, "A high-speed four-transmitter four-receiver MIMO OFDM test-bed: Experimental results and analyses," EURASIP Journal on Applied Signal Processing, vol. 2006, pp. 1-10.

[3] T. Haustein, A. Forck, H. Gabler, V. Jungnickel, and S. Schiffermuller, "Real-time signal processing for multiantenna systems: algorithms, optimization and implementation on an experimental test-bed," EURASIP Journal on Applied Signal Processing, vol. 2006, pp.121.

[4] D. Borkowski, L. Bruhl, C. Degen, W. Keusgen, G. Alirezaei,F. Geschewski, C. Oikonomopoulos, and B.
Rembold, "SABA: A test-bed for a real-Time MIMO system," EURASIP Journal on Applied Signal Processing, vol. 2006, no. 1, pp. 1-15.

[5] J.W. Weijers, V. Derudder, S. Janssens, F. Petr, and A. Bourdoux,"From MIMO-OFDM algorithms to a real time wireless prototype: A systematic Matlab-tohardware design flow," EURASIP Journal on Applied Signal Processing, vol. 2006, pp. 1-12.

[6] K. C. N. Madani and N. Hilton, "An antenna simulator system for satellite communications adaptive digital beam forming network", Recent Advances in Microwave Subsystems for Space and Satellite Applications, London, 1993

[7] Taco Kluwer, KeesSliump, RoelSchiphorst and FokkeHoekesem, "Development of a smart-antenna testbed, demonstrating software defined digital beam forming", Proceedings of the 12th Annual Workshop on Circuits, Systems and Signal Processing, Netherlands, Nov 2001.

[8] Heung-Jae Im, Wonsang HwangSeungwon Choi, Hyeongdong Kim, "Performance analysis of a smart antenna system utilizing a test-bed implemented on a DSP board", Microwave Conference Asia-Pacific, 2000, $\operatorname{pg} 799-803$

[9] Juan Torres-Rosario, "Implementation of a phased array antenna using digital beamforming" MSc thesis Uni. of Puerto

Rico,

2005 